\title{
Descripción Anatómica del Esqueleto de los Tractos Respiratorios Superiores del Periquito Australiano (Melopsittacus undulatus)
}

\author{
Anatomical Description of Upper Respiratory Tracts Skeleton of Budgerigar (Melopsittacus undulatus)
}

\author{
Jorge Ricardo Gersenowies Rodríguez \& Gabriela Sánchez Fabila
}

GERSENOWIES, R. J. R. \& SÁNCHEZ, F. G. Descripción anatómica del esqueleto de los tractos respiratorios superiores del periquito australiano (Melopsittacus undulatus). Int. J. Morphol., 28(1):85-92, 2010.

RESUMEN: Se describe detalladamente los elementos esqueléticos de los tractos respiratorios superiores de 10 periquitos australianos (Melopsittacus undulatus), dicha descripción permitió encontrar claras diferencias en su anatomía no referidas con anterioridad, debido posiblemente al interés por estudiar la siringe y no los tractos respiratorios completos, el objetivo principal fue describir la morfología y osificación de los tractos respiratorios superiores del periquito australiano, a través de la técnica de transparentación con doble tinción (rojo de alizarina "S" y azul de alciano), que permite observar la osificación de los tractos respiratorios; obteniendo como resultado dos diferencias óseas importantes: (1) la lengua exhibe dos pequeños huesos paraglosales asociados con la movilidad de la misma y (2) una importante osificación desde la lengua hasta los elementos "A" siringeales. En cuanto al patrón del esqueleto traqueal y siríngeo, no se observan grandes diferencias con respecto a otros psittaciformes. Se propone el estudio del esqueleto lingual para determinar su importancia en el establecimiento de relaciones de parentesco y su valor taxonómico, y de esta forma, abrir una puerta para nuevos estudios de morfología comparativa.

PALABRAS CLAVE: Esqueleto lingual; Psittacidae; Siringe; Tráquea; Osificación.

\section{INTRODUCCIÓN}

La compresión de los tractos respiratorios superiores en aves es muy importante en el estudio ornitológico, esto en parte es debido a que dichos tractos han evolucionado de forma diferente a la ocurrida en mamíferos. Los cambios filogenéticos han llegado a producir posiblemente el sistema respiratorio más eficiente entre los vertebrados. Algunos de estos cambios se presentan como caracteres que permiten establecer relaciones de parentesco de forma precisa (Morrisey, 1997); por lo que es necesario revisar brevemente el desarrollo de las características anatómicas de los tractos superiores del sistema respiratorio de aves. Desde las primeras investigaciones de las vías respiratorias de las aves, la siringe ha recibido una gran atención debido a su función como órgano fonador, asumiendo la función de la laringe de los mamíferos, hecho por el cual ha sido el centro de múltiples estudios desde el siglo XVII (Owen, 1866; Huxley, 1871). Sin embargo, la laringe, tráquea y siringe desde el punto de vista anatómico parece ser sumamente variables entre las especies de aves.

Los tractos respiratorios superiores en las aves continúan con una única coana en la laringe posterior a la cavidad nasal. La laringe que no posee ningún papel esencial en la producción de sonido, generalmente consiste de cuatro cartílagos que en algunas especies se osifica parcialmente (Fitzgerald, 1970; King \& McLelland, 1984). La tráquea está construida por una serie de cartílagos traqueales que hasta cierto punto, se osifica en varias especies (Piperno \& Peirone, 1975). Se bifurcan los bronquios primarios casi a nivel de la siringe que es la principal responsable de la vocalización de las aves (Fitzgerald; Dyce et al., 1996). Dos pares de delgadas membranas están presentes en la siringe asociadas con la producción de sonidos, las cuales son conocidas como las membranas timpaniformes lateral y medial (Piperno \& Peirone).

Los periquitos australianos pertenecen al orden Psittaciformes que incluyen a los pericos, loros y guacamayas. El estudio de los tractos respiratorio superiores de los Psittaciformes se centra en la siringe, en donde se ha encontrado que su anatomía varía ampliamente, incluso en grupos relativamente pequeños (Ames, 1971; Gaban-Lima \& Höfling, 2006) y los estudios de la anatomía comparada de la siringe han ayudado a esclarecer 
dos problemas; el primero consiste en establecer los mecanismos involucrados en la producción y modulación del canto del ave y el segundo esclarece las relaciones filogenéticas y taxonómicas entre los diversos grupos de aves (Ames; GabanLima \& Höfling).

Resulta interesante que los estudios completos de los tractos respiratorios superiores en aves, dada la relevancia que tiene la bien conocida tendencia de los cartílagos de la laringe y tráquea aosificarse, y de hecho fueron considerados como un carácter de diagnosis por los primero anatomistas de vertebrados (Owen; Huxley). Sin embargo, solo se tiene información para especies particulares de interés avícola.

Pocos trabajos existen sobre el esqueleto lingual de psittaciformes (Beckers et al., 2004), pero es conocido que la gran movilidad de la lengua modula la vocalización dando características independientes de la fuente al sonido que emite (Patterson \& Pepperberg, 1994, 1998; Warren et al., 1996).

White (1970), estudió la anatomía de la laringe del gallo común incluyendo información sobre el tiempo de desarrollo y distribución de la osificación en un grupo de Leghorn Blancos. Posteriormente, White (Sisson \& Grossman, 2002) describió la magnitud usual de osificación en aves adultas.

Actualmente sólo se dispone de descripciones generales sobre la presencia y distribución de la osificación de la tráquea. Se ha descrito que ocurre en los anillos traqueales de especies grandes (King \& McLelland, 1975), o solo en los anillos caudales de muchas especies (Sisson \& Grossman). Pero está bien determinado su ocurrencia en todos los anillos de los géneros Cygnus y en la familia Gruidae (Duncker, 1971). Las referencias específicas a otros tipos de aves son pocas. Garside (1968) reportó la osificación de los anillos traqueales en 200 aves domesticas examinándolas histológicamente, encontrando que la osificación comienza a las 15 semanas en la región caudal de la tráquea y posteriormente se extiende hasta la región craneal. Según Bradley (1960), la osificación solo ocurre en las porciones ventrales de los anillos traqueales.

En la siringe, se ha descrito que los cartílagos que forman el tímpano se osifican (Gross, 1964). Los extremos ventrales del primer cartílago siringeal bronquial fueron descritos por Myers (1917) como osificados en adultos.

Recientemente Cevik-Demirkan et al. (2007) realizaron la descripción de las características histológicas de la laringe, tráquea y siringe de la codorniz japonesa (Coturnix coturnix japonicum). Encontraron un cartílago cricoides triangular, de 83 a 91 anillos traqueales. Mostrando la gran variabilidad de los tractos respiratorios superiores en aves.

El objetivo principal del presente trabajo consistió en describir la osificación del esqueleto de los tractos respiratorios superiores del periquito australiano, y la morfología de las diferentes porciones constituyentes de los tractos respiratorios superiores, en particular el laríngeo y lingual.

\section{MATERIAL Y MÉTODO}

Se utilizaron 10 especímenes de periquito australiano (Melopsittacus undulatus) especie perteneciente al orden Psittaciformes; obtenidos en centros comerciales especializados, los cuales poseían una masa de 33,4+5,2 grs. Los especímenes fueron sacrificados por inyección intraperitoneal de $0,5 \mathrm{ml}$ de pentobarbital sódico. Posteriormente se desplumaron y se fijaron en formaldehido al $4 \%$ por dos días, lavándose en agua corriente por otros dos días.

Al término de dicho tiempo los especímenes completos fueron sometidos a un proceso de tinción diferencial que consiste de los siguientes pasos:

1. Deshidratación en un tren de alcohol etílico (40, 60, 80, 90 y 95\%) dos días en cada solución.

2. Inmersión de en una solución ácida de azul de alciano (que tiñe las estructuras cartilaginosas) la cual consiste de $10 \mathrm{mg}$ de azul de alciano, $80 \mathrm{ml}$ de alcohol etílico $95 \%$ y $20 \mathrm{ml}$ de acido acético glacial, por $24 \mathrm{hrs}$.

3. Rehidratación en un tren invertido de alcohol etílico (absoluto, 95, 90, 80, 60 y 40\%) dos días encada solución.

4. Lavado en agua corriente por un día.

5. Inmersión en una solución alcalina de rojo de alizarina "S" (que tiñe las estructuras calcificadas) y que consisten de $0.1 \%$ de rojo de alizarina "S" en hidróxido de sodio al 4\%, por tres días.

Posteriormente los especímenes fueron sumergidos en una solución de hidróxido de sodio al 4\% para la digestión alcalina de los tejidos suaves, se mantuvo el procedimiento renovando la solución cada 15 días hasta que los especímenes estuvieron perfectamente diafanizados, proceso que llevó en promedio 12 semanas. Se procedió a desollar el organismo y se retiró el exceso de grasa.

Por último, los especímenes se aclararon a través de un tren de glicerina (40, 60, 80, 90 y 100\%), siendo almacenados en glicerina al $100 \%$ (Fig. 1)

Se extrajeron los tractos respiratorios superiores incluyendo la lengua para su descripción (Fig. 2). Las diferentes porciones fueron observadas, fotografiadas y se describieron utilizando un microscopio estereoscópico Motic B1-223a con una cámara digital Moticam 2000 usando el software Motic Image Plus 2.

Consecutivamente las imágenes fueron limpiadas utilizando el software Adobe photoshop versión 7. La nomenclatura utilizada sigue a la Nomina Anatómica Avium (Sisson \& Grossman) mientras que para los elementos esqueléticos de la siringe se empleo la utilizada por Gaban-Lima \& Höfling la cual a su vez está basada en Ames.

\section{RESULTADOS}

Los resultados se presentan ordenados en las distintas regiones consideradas: 

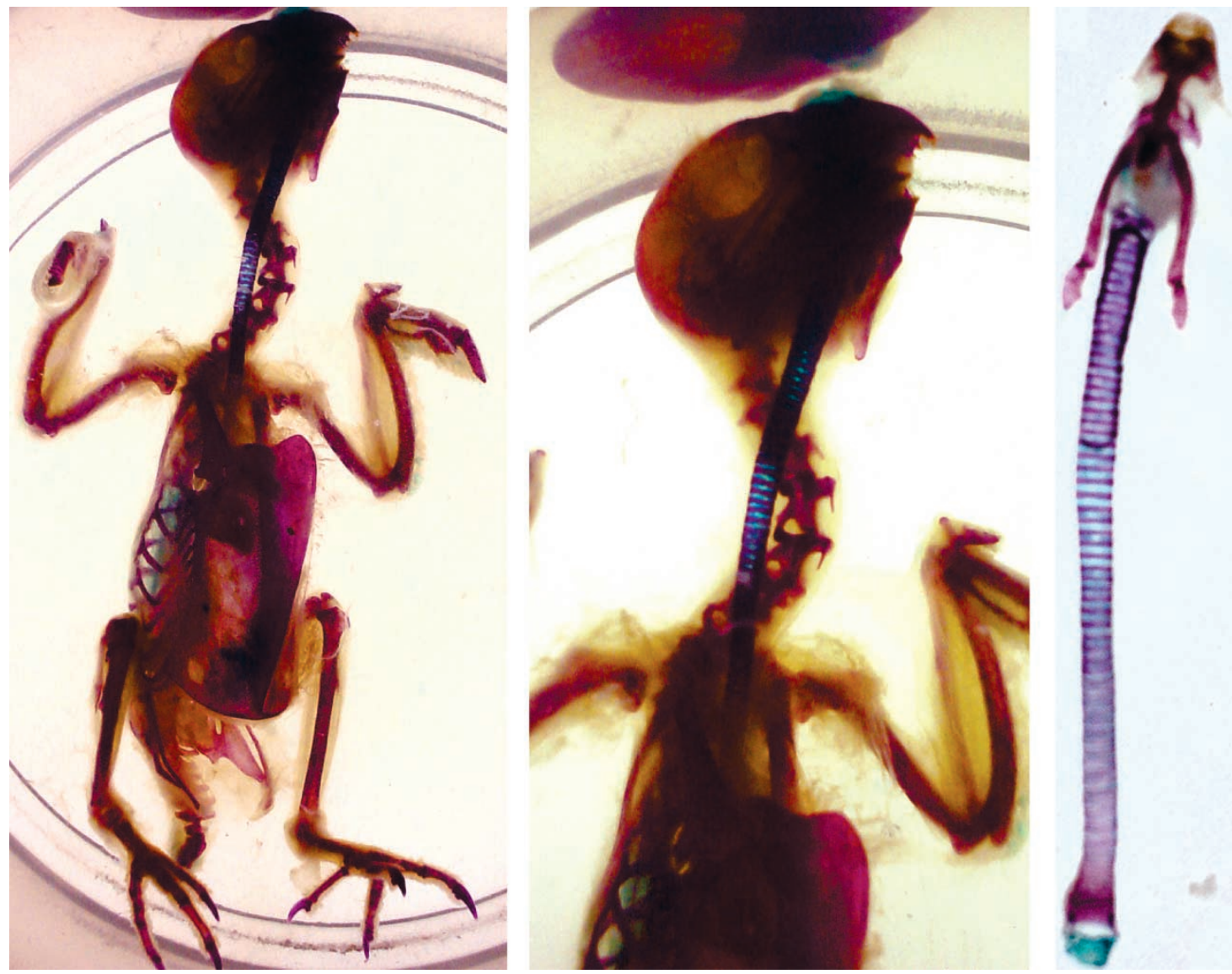

Figura 1. Espécimen transparentado de periquito australiano (Melopsittacus undulatus). A. Periquito transparentado vista ventral. B. Acercamiento en la región donde se observa el tracto respiratorio superior. C. Tracto respiratorio superior disecado.

Esqueleto lingual. El esqueleto lingual está formado por las partes paraglosal (entoglossal) y basibranquial rostral (basihyal) del hueso hioides teniendo una longitud rostrocaudal de $13,79 \mathrm{~mm}$ (Fig. 2).

El hueso paraglosal tiene una forma rectangular con un longitud rostrocaudal de $2,32 \mathrm{~mm}$ con un ancho mayor de 0,83 $\mathrm{mm}$ y un ancho menor de $0,32 \mathrm{~mm}$. Se encuentra ubicado en posición paramedial, es par y se asienta en la parte libre de la lengua. A partir del extremo cefálico se extiende rostrolateralmente un par de cuernos cortos paraglosales cefálico, mientras que en el extremo caudal un par de cortos cuernos de huesos paraglosales se extienden caudolateralmente. Caudalmente el hueso paraglosal forma una articulación sinovial poliaxial con el hueso basibranquial rostral (Figura 2), esto implica que el hueso paraglosal goza de una gran movilidad en muchos planos.

El hueso basibranquial rostral se asienta en la parte fija de la lengua, sus extremos rostral posee un área articular esférica mientras que la caudal es relativamente ancha sin movilidad. Posee una longitud rostrocaudal de 4,42 $\mathrm{mm}$ con un ancho mayor de 0,95 $\mathrm{mm}$ y un ancho menor de 0,32 $\mathrm{mm}$. En su porción lateral se obser- va un par de huesos adicionales no descritos con anterioridad, esto se denominaron de acuerdo a su posición parabasibranquiales el cual posee una longitud rostrocaudal de $1,05 \mathrm{~mm}$ con un ancho de $1,47 \mathrm{~mm}$. Presentan en su superficie dorsal un arco muy bien desarrollada que posee una dirección rostrocaudal que posee una base de $2 \mathrm{~mm}$ con una altura de $1,47 \mathrm{~mm}$, el arco posee un extremo apical plano de $0,42 \mathrm{~mm}$. También es notoria una placa que se extiende lateralmente, esta se denominó ala caudolateral del parabasibranquial (Figura 2).

Caudalmente, se articula con el hueso basibranquial caudal en posición medial que posee una longitud rostrocaudal de 1,89 $\mathrm{mm}$ con un ancho mayor de $0,74 \mathrm{~mm}$ y un ancho menor de 0,22 mm (Fig. 2).

El basibranquial rostral se articula lateralmente con el ceratobranquial, el cual es un hueso largo con una longitud rostrocaudal de $6,32 \mathrm{~mm}$, un ancho mayor de $0,84 \mathrm{~mm}$ y un ancho menor de $0.42 \mathrm{~mm}$. El ceratobranquial caudalmente se articula con los ceratobranquiales que son muy cortos y con forma de almendra, poseen una longitud rostrocaudal de $2,11 \mathrm{~mm}$ con una anchura máxima de 1,05 mm (Fig. 2). Con excepción del hueso paraglosal 


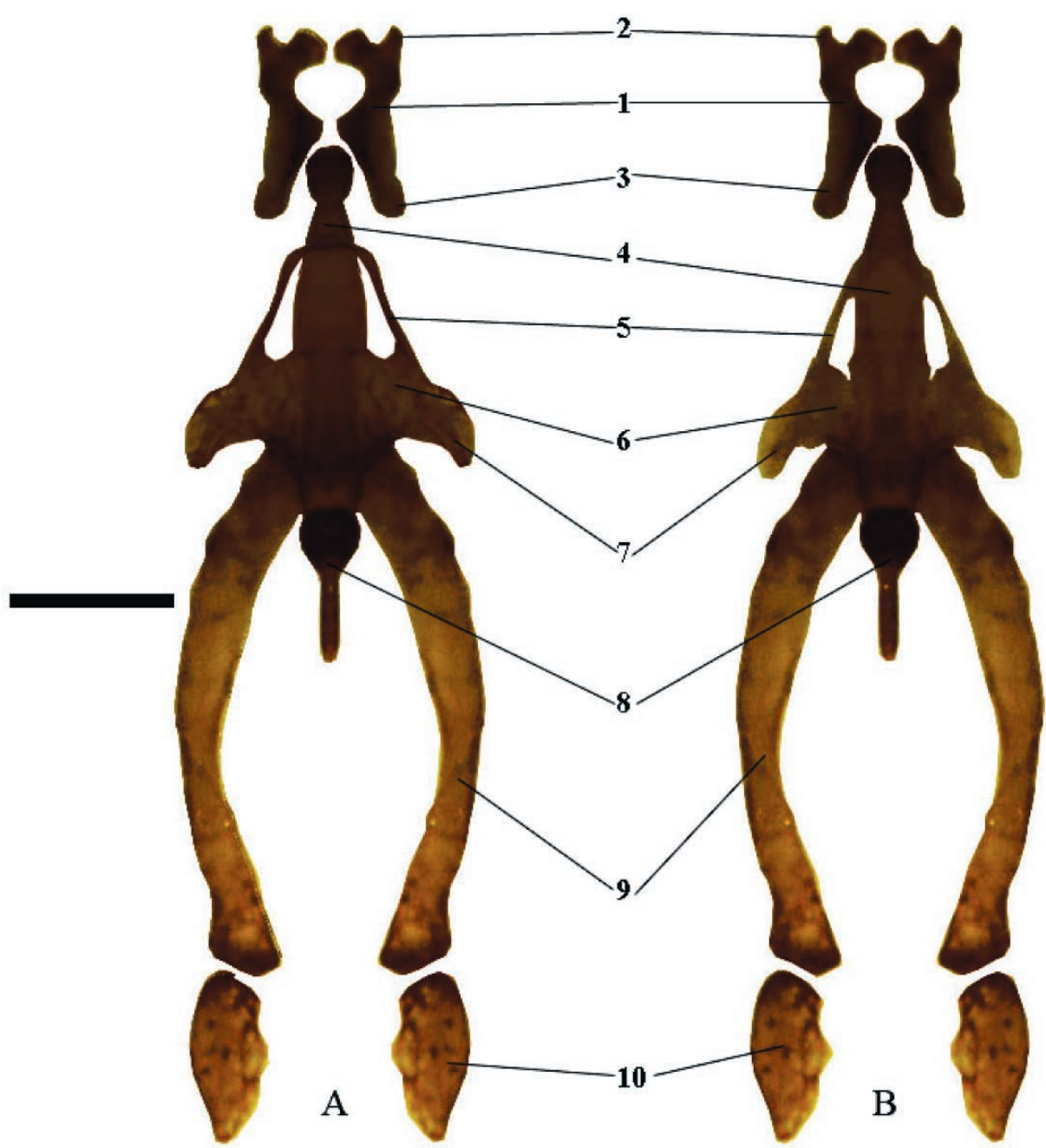

Fig. 2. Esqueleto lingual del periquito australiano. A.- Vista dorsal; B.- Vista ventral. , 1.- hueso paraglosal (entoglossal); 2. Cuerno cefálico del hueso paraglosal; 3. Cuerno caudal de hueso paraglosal; 4. Basibranquial rostral (basihyal); 5. Arco dorsal de los parabasibranquiales; 6. Parabasibranquiales. 7. Ala del parabasibranquial; 8. Basibranquial caudal (urohyal); 9. Ceratobranquial; 10.Hueso epibranquial. Longitud de la barra $=20 \mathrm{~mm}$.

y de los huesos parabasibranquiales, los restantes elementos posee el patrón básico descrito por Sisson \& Grossman (2002) para las aves en general.

Esqueleto laríngeo: El esqueleto laríngeo está formado por cuatro elementos, el cricoides medial, el procricoides y los aritenoides pares.

El cricoides es impar (Fig. 3). Es el componente mayor y posee unos 5,15 $\mathrm{mm}$ de longitud rostrocaudal y $6,03 \mathrm{~mm}$ de ancho en su parte más ancha que es la caudal. Posee formas de herradura. Está constituido por tres componentes: a) Placa cricoides ventral (Figura 3), es una placa larga, cóncava dorsalmente. Esta completamente osificada, no observándose la expansión rostral típica de otras aves. Las alas izquierda y derecha (láminas cricoides dorsales) están fusionadas con la porción lateral del cuerpo. El borde medial de cada ala del cricoides se une por una articulación sinovial con el procricoides y así se forman la articulación procricocricoidea. El borde lateral del ala es algo más grueso y se adelgaza hacia su contacto con el borde rostral de la placa cricoides ventral. No se observa una articulación directa entre el cricoides y el aritenoides. $\mathrm{Ni}$ se observan porciones cartilaginosas en el cricoides.

El procricoides es medial, dorsal y pequeño (Figura 3). Tiene forma de rectangular. Posee una anchura de 2,19 $\mathrm{mm}$ con una longitud rostrocaudal de $0,82 \mathrm{~mm}$. En la zona dorsolateral rostral posee las áreas articulares donde se unen los cuerpos del aritenoides izquierdo y derecho, formando así la articulación procricoaritenoidea. Caudalmente posee otras dos áreas articulares izquierda y derecha, en las cuales se unen las alas cricoideas izquierda y derecha (articulación procricocricoidea). El procricoides está completamente osificado. 


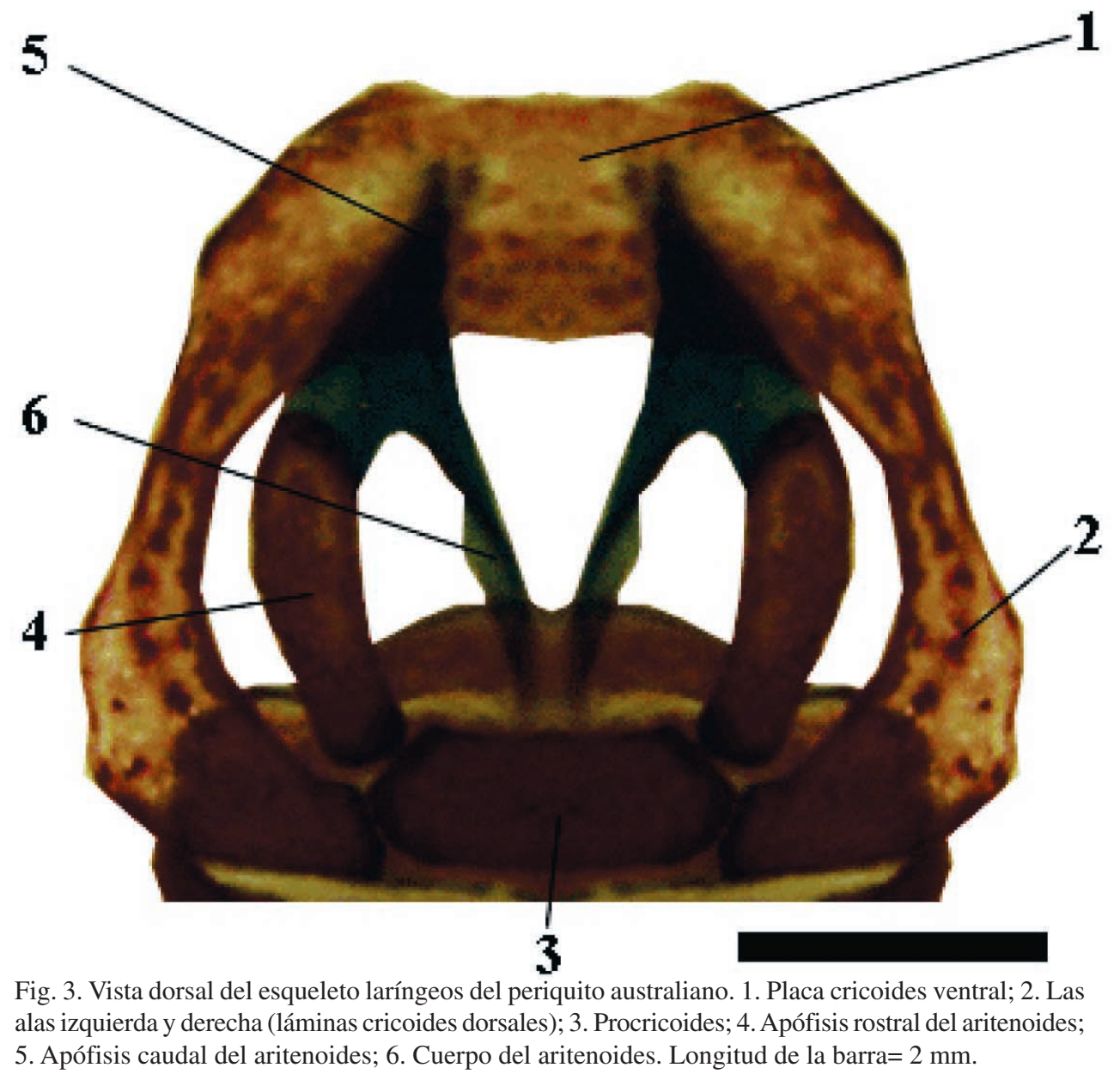

Los aritenoides son pares (Fig. 3). Cada uno está formado por un cuerpo que posee una longitud rostrocaudal de $2,3 \mathrm{~mm}$ y un ancho de $0,71 \mathrm{~mm}$, una apófisis rostral de 1,75 de longitud rostrocaudal y una apófisis caudal con 1,97 de longitud rostrocaudal. El cuerpo se articula caudomedialmente con el procricoides mediante una articulación sinovial, llamada articulación procricoaritenoidea. El cuerpo también se desliza libremente sobre el borde medial del ala cricoides. Únicamente el cuerpo del aritenoides está completamente osificado.

Esqueleto traqueal: Los elementos traqueales varían de 59 a 68 anillos, en cuanto a número, con osificación en su cuerpo central y zonas cartilaginosas en sus extremos rostral y caudal (Fig. 4). El primero sigue inmediatamente después de cartílago cricoides y al menos uno precede al tímpano de la siringe. Cada anillo es un círculo completo (Figura 4). No hay un solapamiento entre los anillo en ninguna de sus porciones. El diámetro, progresivamente, decrece (Figura 4). Así, en los anillos más anchos se encuentran en el tercio rostral de la tráquea, con un diámetro promedio de $3,68 \mathrm{~mm}$ y una anchura promedio de $0,74 \mathrm{~mm}$; En el tercio intermedio se observó un diámetro promedio de $2,41 \mathrm{~mm}$ y una anchura promedio de 0,64 $\mathrm{mm}$. Los anillos del tercio siringeal poseían un diámetro promedio de $1,98 \mathrm{~mm}$ y una anchura promedio de $0,48 \mathrm{~mm}$.
Esqueleto siringeal: La siringe de 47 especies de Psittaciformes han sido descritas exhaustivamente, este órgano está situado en la bifurcación de la tráquea, penetra en los bronquios derecho e izquierdo, precisamente en el interior del orificio celómico y queda suspendido en el seno del saco aéreo clavicular. La siringe posee una longitud rostro caudal de $8.96 \mathrm{~mm}$, con un ancho mayor de $3,94 \mathrm{~mm}$ y un ancho menor de $1,93 \mathrm{~mm}$. Su característica externa más notable es un acentuado incremento de la longitud dorsoventral. El esqueleto está compuesto por cuatro elementos.

Los elementos craneales o elementos "A" (Fig. 5), que se componen de cuatro anillos (A2 al A5) y dos elementos pares (A1). $\mathrm{Su}$ diámetro es ligeramente mayor que en los anillos traqueales precedentes; así mismo se hallan más cercanos de modo que en algunos puntos sus bordes dorsales se funden y en otros se hallan unidos por tejido fibroso denso. Según Myers son de origen traqueal (Sisson \& Grossman).

Los elementos craneales se dividen con claridad, cranealmente en el tympanum (Fig. 5), un cilindro formado por la fusión parcial o total de 4 elementos "A" completos que adquieren una forma del anillo. Los procesos ventrales y dorsales del tímpano se extienden fuera del borde caudal con una forma aproximada- 
A

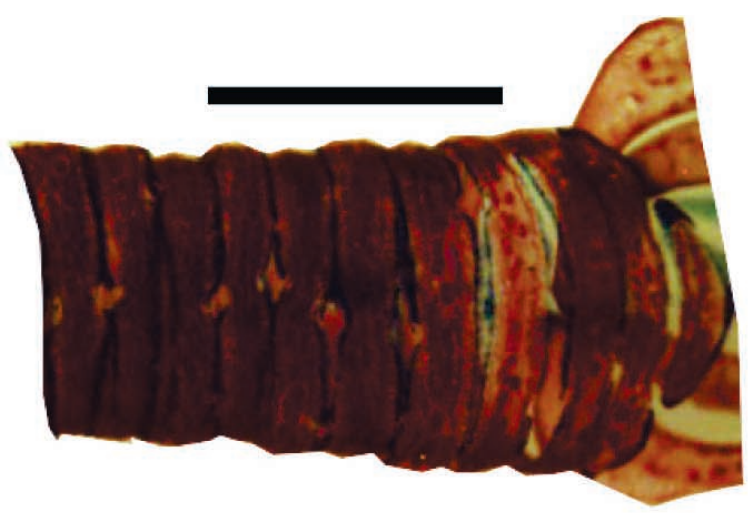

B

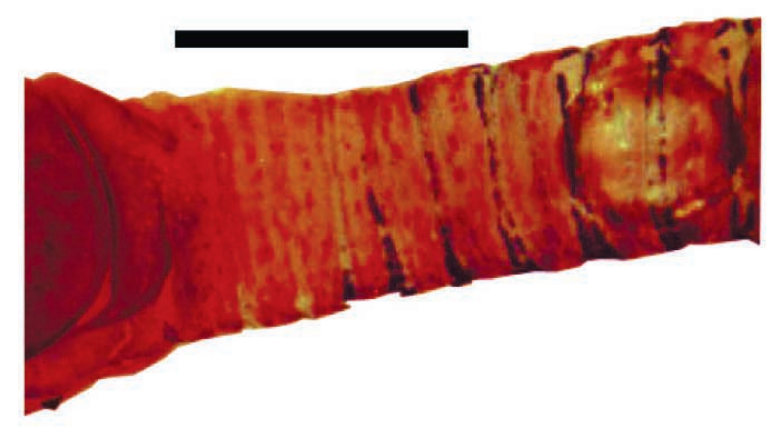

$\mathrm{C}$

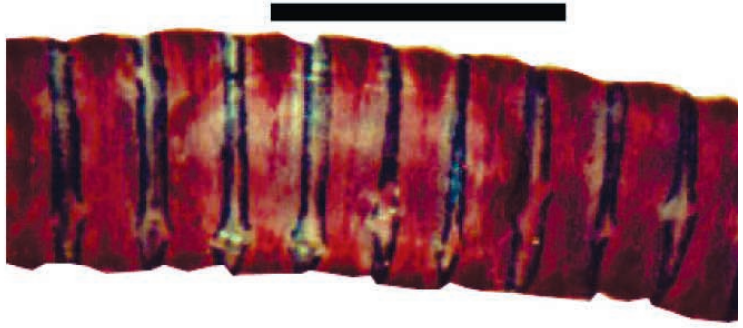

Fig. 4. Vistas laterales de la tráquea del periquito australiano.

A. Porción rostral.

B. Porción intermedia.

C. Porción caudal.

Longitud de la barra $=2 \mathrm{~mm}$. mente triangular conocido como proceso timpaniforme ventral y dorsal (Fig. 5). Ambos procesos se unen medialmente dividiendo la luz de la tráquea en los dos bronquios, los dos procesos unidos en conjunto constituyen el Pessulus.

Caudalmente al tympanum y unido a él hay un par de proyecciones esqueléticas planas en posición lateral a la tráquea y soportando la porción craneal de la Mem. tympaniformis lateralis, de ahí el termino de placas timpánicas (A1) (Fig. 5). La Mem. tympaniformis lateralis (Fig. 5) son soportadas cranealmente por las placas timpánicas y caudalmente por el elemento B1.

Las placas timpánicas se interpretan según Gaban-Lima \& Höfling como el elemento "A1" (Fig. 5) mediamente se divide en dos pares de procesos ventrales y dorsales. Los procesos ventrales y dorsales de la placa timpánica se extienden fuera de su borde caudal, tanto sobre el vértice ventral como por el dorsal (Fig. 5). En esta placa se delimitan dos regiones (Fig. 5): (1) Gruesa y esponjosa, similar a los elementos "A" que constituyen parte del tympanum, ocupando la porción más craneal; (2) los procesos caudales y sus bordes ventrales y dorsales, cuyos extremos es cartilaginoso.

Los elementos "B" siempre son pares y medialmente incompleto (Fig. 5), se encuentran en número de 5 a 6 . Los elementos B más craneales son generalmente cóncavos y tienen una región lateral plana que se une a las placas timpánicas. En dirección caudal estas regiones adelgazan, junto con la concavidad craneal, hasta que ellos desaparecen. Los elementos más caudales se tienden a ser más delgados (Fig. 5).

\section{DISCUSIÓN}

El esqueleto lingual, es la estructura que presenta las variaciones más interesantes, la presencia de un par de huesos paraglosales que se unen al resto del esqueleto lingual a través de la articulación poliaxial del basibranquial le permiten una amplia movilidad a la lengua, lo cual está de acuerdo por lo reportado por diferentes autores (Patterson \& Pepperberg, 1994, 1998; Warren et al.) que indica que dicha propiedad permite a la lengua modular la vocalización dando características independientes de la fuente al sonido que emite. Las características de los paraglosales no tienen semejanza con los esqueletos linguales reportados para galliformes, carpinteros y algunas paseriformes donde se ha descrito como un hueso impar (Sisson \& Grossman). También hay que mencionar que el arco del parabasibranquial es una estructura que sirve de inserción a una gran cantidad de músculos lo cuales se pueden asociar con la gran movilidad de la lengua. Este carácter, en caso de demostrarse su existencia en otros psitaciformes, consideramos que puede tener una gran importancia, tanto para el establecimiento de relaciones de parentesco entre las diferentes especies de pericos, como sus adaptaciones a su ambiente.

El esqueleto laríngeo muestra varias diferencias con respecto a lo reportado en diferentes especies (Sisson \& Grossman), en particular hay dos caracteres que sobresalen, la gran osificación de los elementos constituyentes y la falta de la expansión rostral típica de otras aves. Estas características no indican que es una estructura mucho más rígida, lo cual está en relación directa con 


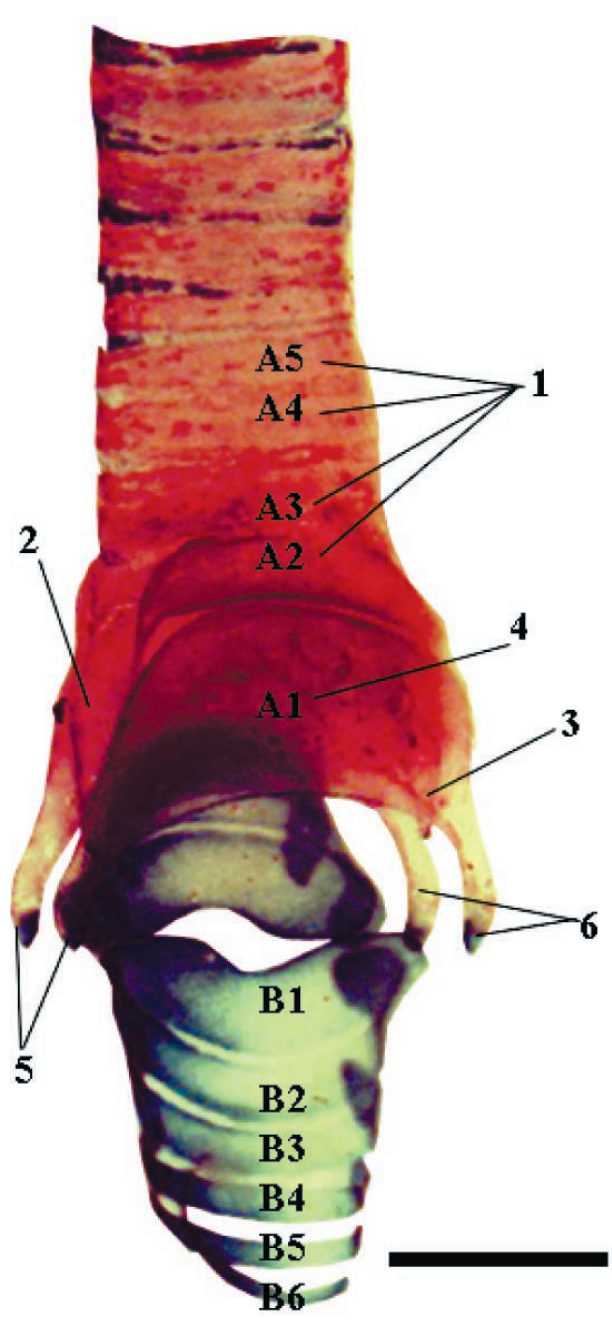

la resistencia necesaria para soportar la gran musculatura asociada con la movilidad de la lengua.

Los anillos traqueales muestra las características típicas del grupo de las psitaciformes que ya han sido reportadas (GabanLima \& Höfling), sin embargo, se observa una gran calcificación central en todos los anillos traqueales, posiblemente se deba a que solo se utilizaron especímenes adultos.

La siringe muestra las características típicas de los psitaciformes ya descrita por otros autores (Gaban-Lima \& Höfling), indicando que es un carácter constante dentro de este grupo.

De acuerdo a nuestros resultados podemos extraer las siguientes conclusiones:

1. La osificación desde la lengua hasta las elementos "A" de la siringe fue un carácter constante en los tractos respiratorios superiores en este tipo de ave. De ahí que la creencia sostenida algunos autores (King \& McLelland, 1975) en relación que hay un predo-

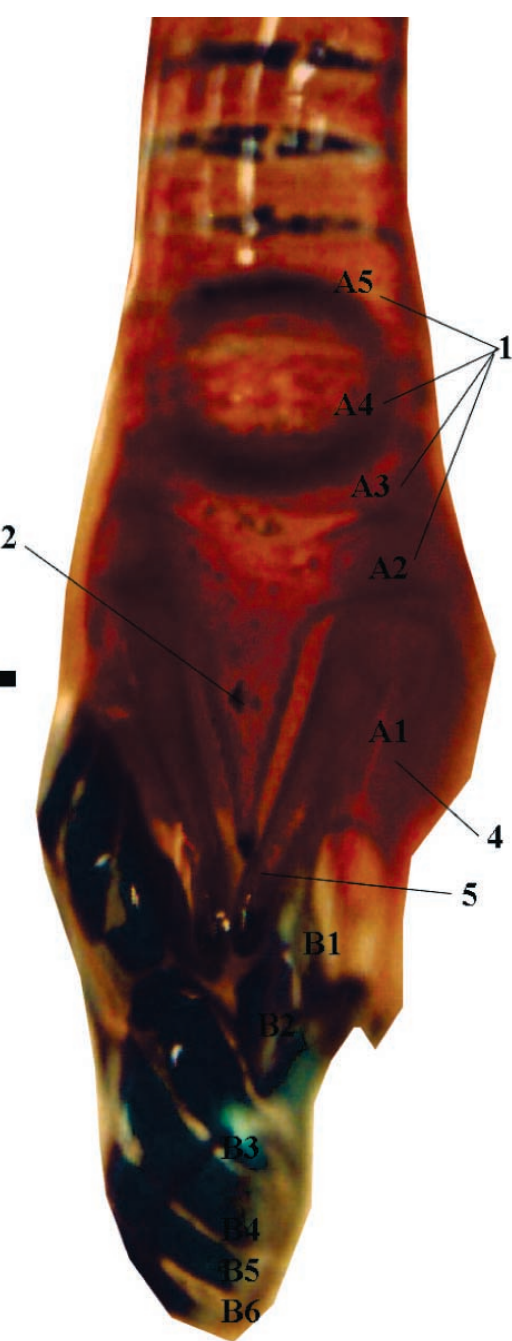

Fig. 5. Esqueleto siringeal del periquito australiano. A. Vista lateral. B. Vista ventral. 1. Tympanum; 2.- Proceso timpaniforme ventral; 3.- Proceso timpaniforme dorsal; 4. Placas timpánicas; 5. Procesos ventrales; 6 . Procesos dorsales. "A1" a "A5" elementos craneales o elementos "A". "B1" a "B5" elementos "B". Longitud de las barras $=2$ $\mathrm{mm}$.

minio de la osificación solo en especies grandes, no se comprueba en esta especie pequeña.

2. Se encontró una estructura lingual y laríngea muy particular, la cual está asociada con una gran movilidad lingual previamente reportada por diferentes autores (Patterson \& Pepperberg, 1994, 1998; Warren et al.). Se propone que este carácter puede tener importancia tanto para establecer relaciones de parentesco, como para el análisis de sus adaptaciones al ambiente entre las diferentes especies de pericos, sin embargo, esto solo se puede probar con una mayor cantidad de estudios en otras especies de psittaciformes.

3. Con respecto al patrón del esqueleto traqueal y faríngeo, no se observan grandes diferencias con respecto a la información ya publicada (Gaban-Lima \& Höfling).

AGRADECIMIENTOS. Agradecemos a los integrantes del laboratorio de microscopía óptica y fotografía digital de la FES Iztacala, dirigidos por el Biol. Héctor Barrera Escorcia, su apoyo en la captura digital de imágenes. 
GERSENOWIES, R. J. R. \& SÁNCHEZ, F. G. Anatomical description of upper respiratory tracts skeleton of budgerigar (Melopsittacus undulatus).Int. J. Morphol., 28(1):85-92, 2010.

SUMMARY: In this work describes in detail skeletical elements of upper respiratory tracts of 10 budgerigars (Melopsittacus undulatus), the description obtains evident differences in its anatomy doesn't relate before, this should to be possible at interest to study syrinx and not full respiratory tracts the objective was to describer morphology and ossification of full upper respiratory tracts of budgerigar, this obtained utilizing clearing technique's with stain double (alizarin red "S" and alcian blue) it allows to observe ossification of respiratory tracts, it obtained like result two principal evident differences: (1) the lingual shows two small paragloss bones associates with its mobility and (2) a important ossification from the lingual to "A" syrinx elements. Respectful at patron tracheal and syrinx skeletical doesn't show evident differences in comparation with other psittacidae. It proposes a study of lingual skeletical to determination of importance for to establish relationship and its taxonomic value and this form meets a door for new studies of comparative morphology.

KEY WORDS: Lingual skeleton; Psittacidae; Syrinx; Trachea; Ossification.

\section{REFERENCIAS BIBLIOGRÁFICAS}

Ames, P. L. The morphology of the syrinx in passerine birds. Peabody Museum Natural History Yale University, 37: 1-194, 1971.

Beckers, G. J. L; Brian, B. S; Nelson, S. \& Suthers, R. A. VocalTract Filtering by Lingual Articulation in a Parrot. Curr. Biol., 14:1592-7, 2004.

Bradley, O. C. The Structure of the Fowl. $4^{\text {th }}$ ed. Edinburgh and London, Oliver and Boyd, 1960. p.139.

Cevik-Demirkan, A.; Hazirog `lu, R. M. \& Kürtül, I. Gross Morphological and Histological Features of Larynx, Trachea and Syrinx in Japanese Quail. Anat. Histol. Embryol., 36:2159, 2007.

Duncker, H. R. The lung air sac system of birds. Adv. Anat. Embryol. Cell Biol., 45:1-171, 1971.

Dyce, K. M.; Sack, W. O. \& Wensing, C. J. G. Textbook of Veterinary Anatomy. $2^{\text {nd }}$ edn. Philadelphia, W.B. Saunders Co., 1996. p.856.

Fitzgerald, C. T. The Coturnix Quail Anatomy and Histology. Ames, University Press, 1970. p.306.

Gaban-Lima, R. \& Höfling, E. Comparative anatomy of the syrinx in the tribe arini (Aves: Psittacidae). Braz. J. Morphol. Sci., 23(3-4):501-12, 2006.

Garside, J. S. Ossification of the tracheal cartilages in the fowl. Vet. Rec., 82:470-1, 1968.

Gross, W. B. Voice production in the chicken. Poultry Sci., 43:10058, 1964.

Huxley, T. H. A Manual of the Anatomy of Vertebrates Animals. London, J. \& A. Churchill, 1871. p.589.

King, A. S. \& McLelland, J. Outlines of Avian Anatomy. London, Bailliere Tindall, 1975. p.154.
King, A. S. \& McLelland, J. Respiratory system. In: Birds: their Structure and Function. $2^{\text {nd }}$ ed. London, Bailliere and Tindall, 1984. pp.110-4.

Morrisey, J. K. Diseases of the Upper Respiratory Tract of Companion Birds. Sem. Avian Exotic Pet Med., 6:195-200, 1997.

Myers, J. A. Studies on the syrinx of Gallus domesticus. J. Morph., 29:165-215, 1917.

Owen, R. The Anatomy of Vertebrates. London, Longmans Green, 1866. pp. 217-9.

Patterson, D. K \& Pepperberg, I. M. A comparative study of human and parrot phonation: Acoustic articluatory correlates of vowels. J. Acoust. Soc. Am., 96:634-48, 1994.

Patterson, D. K \& Pepperberg I. M. Acoustic and articulatory correlates of stop consonants in a parrot and a human subject. $J$. Acoust. Soc. Am., 103:2197-15, 1998.

Piperno, E. \& Peirone, S. Morphological characteristics and mutual relationships of the tracheal cartilaginous rings in Gallus gallus. Anat. Histol. Embryol., 4:172-8, 1975.

Sisson, S. \& Grossman, J. D. Anatomía de los animales domésticos. Madrid, Salvat S. A., 2002.

Warren, D. K.; Patterson, D. K. \& Pepperberg, I. M. Mechanisms of American English vowel production in a Grey parrot (Psittacus erithacus). Auk, 113:41-58, 1996.

White, S. S. The larynx of Gallus domesticus. Ph. D. Thesis, University of Liverpool, 1970. p.210.

Dirección para correspondencia:

Jorge Ricardo Gersenowies Rodríguez

Laboratorio de anatomía animal comparada.

Unidad de Morfología y Función.

Facultad de Estudios Superiores Iztacala.

Av. de los Barrios \# 1. Col. Los Reyes Iztacala. C. P. 54090 Tlalnepantla, Estado de México - MÉXICO

Email: morfofisiologia@hotmail.com $\begin{aligned} & \text { Recibido :09-07-2009 } \\ & \text { Aceptado:12-01-2010 }\end{aligned}$ 